\title{
Kombinierung eines Wasser-Sensorsystems mit einem vollautomati- schen indirekt kompetitiven Durchfluss-Microarray-Immunoassay als Frühwarn-System für vermehrtes Algenwachstum und Freisetzung von Algentoxinen in Oberflächengewässern
}

\author{
Andreas Auernhammer ${ }^{1}$, Cindy Dongxin $\mathrm{Hu}^{2}$ und Michael Seidel ${ }^{1}$ \\ ${ }^{1}$ Institut für Wasserchemie und chemische Balneologie, TU München, Garching, Deutschland \\ ${ }^{2}$ A.U.G. Signals Ltd., Ontario, Canada \\ Kontakt: a.auernhammer@tum.de
}

\section{Einleitung}

Algenblüten sind sprunghafte und massenhafte Vermehrungen von Cyanobakterien. Begünstigt durch den Klimawandel und der Eutrophierung der Gewässer, wurde dieses Phänomen in den letzten Jahren immer häufiger beobachtet. ${ }^{[1]}$ Ein exponentieller Wachstumsschub der Cyanobakterien trübt nicht nur das Wasser, sondern hat auch einen negativen Einfluss auf aquatische Makrophyten und Lebewesen. Während der Algenblüte steigt die Belastung der Gewässer mit Cyanotoxinen, wie z. B. Microcystin-LR, die schädliche Wirkungen auf das betroffene Ökosystem haben und eine Gefährdung für die menschliche Gesundheit darstellen.

Zur Vorbeugung von Gefährdungen wird in der Badewasserrichtlinie des Europäischen Parlaments vorgeschrieben, dass für Badegewässer eine geeignete Überwachung durchzuführen ist, dessen Profil auf eine mögliche Algenblüte hinweisen und angemessene Bewirtschaftungsmaßnahmen ergriffen werden müssen, sobald eine Massenvermehrung oder potentielle Gesundheitsgefährdung festgestellt wurde. ${ }^{[2]}$ Das Gefahrenpotential wird meistens durch visuelle Prüfung eingeschätzt wobei die Trübung des Wassers, Sichttiefe, Bildung von Schlieren und Aufrahmungen berücksichtigt werden. Im Fall einer Potentiellen Gefahr durch Cyanotoxine geben diskrete Probennahmen mit anschließenden mikroskopischen-, chromatographischen- oder immunologischen Analysen Aufschluss über die Art der anwesenden Cyanobakterien und Cyanotoxinkonzentrationen.

Trotz dieser Vorsichtsmaßnahmen bleiben Algenblüten regelmäßig unentdeckt, bis Unfälle passieren und anschließend erst Cyanobakterien in Verdacht geraten. Bei diesen Unfällen kommen oft Hunde zu schaden, welche Vergiftungserscheinungen zeigen oder an Cyanotoxinvergiftungen sterben. Alleine in diesem Jahr gab es einige Meldungen dieser Art z.B. ist am Tegeler See ${ }^{[3]}$ und am Mandichosee ${ }^{[4]}$ jeweils ein Hund und am Asbachdelta sind fünf Hunde wegen Cyanotoxinvergiftung gestorben ${ }^{[5]}$. Diese unentdeckten Algenblüten stellen nicht nur ein hohes Gesundheitsrisiko für Hunde dar, sondern auch in extremen Fällen für badende Kinder und Erwachsene.

Um solche Unfälle zu vermeiden, ist es zwingend notwendig ein Frühwarnsystem für drohende Algenblüten zu entwickeln, welches in der Lage ist, anhand von Veränderungen bestimmter Parameter, welche eine Algenblüte vorhersagen können, eine drohende Massenvermehrung zu erkennen. Mit einer Cloud-basierten Datenverarbeitung und künstlicher Intelligenz könnte zukünftig Daten in Echtzeit verarbeitet werden und softwaregsteuert eine Warnmeldungen erzeugt werden. Für den Lernalgorithmus ist es wichtig, dass eine direkte Korrelation mit den sensorischen Messdaten und den Konzentrationen an freien und zellgebundenen Cyanotoxinen hergestellt wird. Dafür soll das TRITONWassersensor-System mit einem DurchflussMicroarray-Immunoassay kombiniert werden. Für eine kontinuierliche Messung der Cyanotoxine, ist es wichtig, dass eine kontinuierliche Aufkonzentrie-

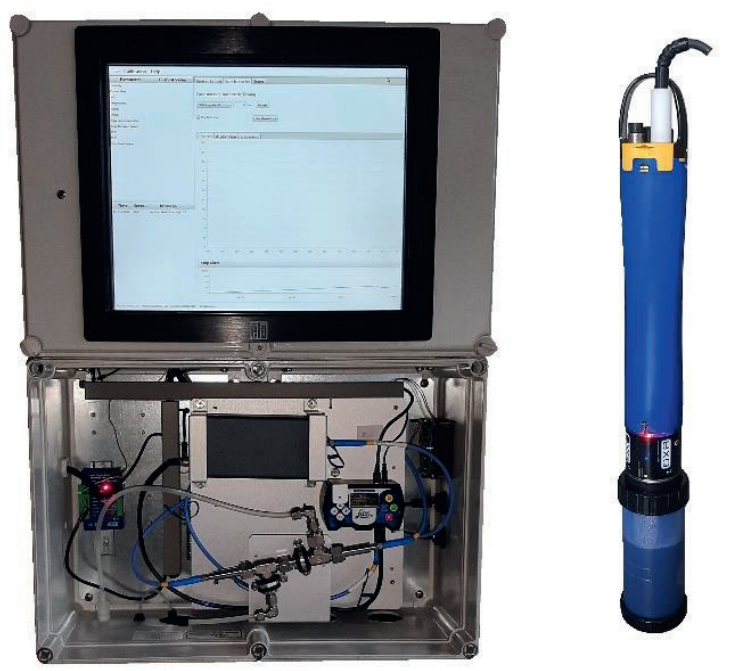

Abb. 1: TRITON-Wassersensor System;

Links oben: Computermodul; Links unten: Optisches Sensormodul; Rechts: EXO-Sensor. 
rungsmethode für freie Cyanotoxine entwickelt wird. Dies soll mittels immunomagnetischer Separation (IMS) ermöglicht werden.

\section{Methoden und Materialien}

Das TRITON-Wassersensor-System ist ein Prototyp, bereitgestellt von A.U.G. Signals Ltd, Toronto, Kanada. Es besteht aus drei Hauptkomponenten, darunter ein Computermodul, ein optisches Sensormodul, welches eine UV-VISAbsorptionsspektralanalyse liefert und eine Multiparametersonde (Exo2 von YSI, Xylem Inc), die $\mathrm{pH}$, Temperatur, Leitfähigkeit, TDS und TSS, Trübung, gelösten Sauerstoff sowie Nitrat und Gesamtstickstoff messen kann. Die EXO-Sonde liefert alle 10 Minuten für jeden Parameter einen Datenpunkt. Das optische Sensormodul ist in der Lage alle 30 Sekunden ein gesamtes Spektrum von 200 bis $850 \mathrm{~nm}$ aufzunehmen. Das wartungsarme TRITONWassersensor-System kann vollständig automatisiert Daten sammeln und über Internetzugriff überwacht werden. Die gesammelten Daten werden in einer online-Datenbank gespeichert und können in Echtzeit ausgewertet werden.

Das physikalische Sensorsystem wird mit einem Biosensor-Analysesystem kombiniert, welches in automatisierter Form Cyanotoxine, wie das Microcystin, Anatoxin oder Saxitoxin, vollautomatisch bestimmt. Das Analyseprinzip beruht auf einen flussbasierten Chemilumineszenz-MikroarrayImmunoassay. Für die Cyanotoxine wird ein indirekt kompetitiver regenerierbarer MikroarrayImmunoassay (Abb. 2) angewendet, der gleichzeitig verschiedener Cyanotoxine innerhalb 7 Minuten

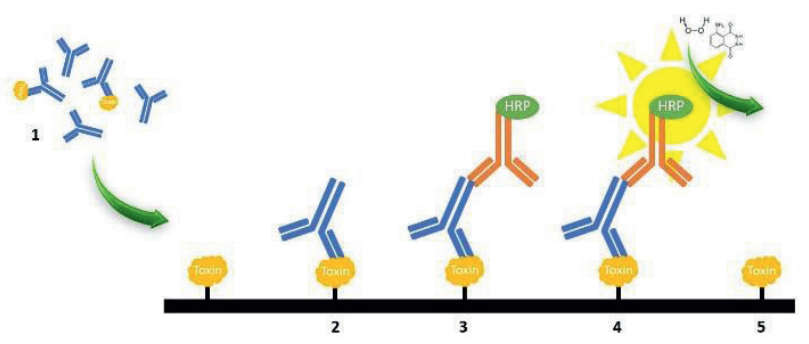

Abb. 2: Schematische Darstellung des indirekt kompetitiven Durchfluss-Microarray Immunoassays. 1) Vorinkubation der Probe mit primären Antikörpern. 2) Binden der noch ungebundenen primären Antikörper an immobilisierte Antigene. 3) Binden der sekundären HRP-

Markierten Antikörper an primäre Antikörper. 4) Chemilumineszenz Reaktion und Bildaufnahme. 5) Regeneration des Microarrays.

quantifizieren kann.

Der Microarray besteht aus einem schwarzem PMMA-Träger mit Ein- und Auslassbohrungen, einer doppelseitigen Klebefolie mit eingestanzter Flusszelle und einem Objektträger, auf dem Toxine immobilisiert sind. Die Oberflächen der Objektträger werden zunächst gesäubert, oxidiert, mit GOPTS silanisiert und anschließend mit einer Polyetheramin (Jeffamine ED-2003) Monolage beschichtet. ${ }^{[6]}$ Anschließend werden die terminalen Aminogruppen der JeffamineSchicht mit Traut-Reagenz behandelt. An die so erzeugten terminalen Thiol-Gruppen kann das MC-LR durch eine Michael-analoge Addition mit der vinylogenen Carbonylgruppe an die modifizierte Chipoberfläche binden. ${ }^{[7]}$ Die Microcystinlösung wird durch Mikrokontaktdruck-Verfahren auf die Chipoberfläche gebracht, wobei jeweils 5 Punkte in einer Reihe erzeugt werden. Durch dieses Verfahren können auch mehrere Reihen verschiedener Cyanotoxine auf dem Microarray immobilisiert werden. Während der vollautomatisierten Messung am MCR-R (Abb. 3) wird zunächst die Probe mit dem primären Antikörper inkubiert und anschließend durch die Flusszelle des Microarrays geleitet, wobei noch ungebundenen Antikörper an das immobilisierte MC-LR binden. Im nächsten Schritt wird der sekundäre Meerrettichperoxidase-markierte Antikörper durch die Flusszelle geleitet und bindet an die bereits immobilisierten primären Antikörper. Durch Injektion einer Mischung von Wasserstoffperoxid und Luminol wird an der Meerrettichperoxidase eine ChemilumineszenzReaktion (CL) katalysiert und mit Hilfe einer CCD-

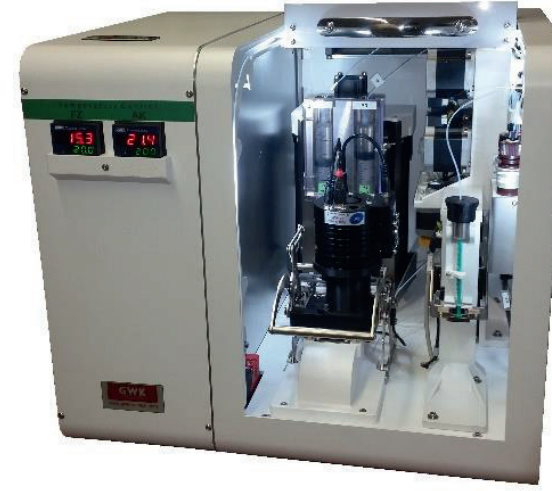

Abb. 3: Microarray-Chip-Reader-R (MCR-R)

Kamera detektiert. Da es sich um einen indirekt kompetitiven Immunoassay handelt, ist die detektierte Signalintensität bei Blindmessungen am höchsten und sinkt mit steigender MC-LR Konzentration.

Wenn die Konzentration von MC-LR das Detektionslimit des Immunoassays unterschreitet, ist eine Aufkonzentrierung der Toxine notwendig. Dafür eignet sich die IMS. Dabei werden Fängerantikörper an magnetische Nanopartikel gebunden und mit der Probe inkubiert. Anschließend können die magnetischen Nanopartikel mit einem Magneten separiert werden, in einem geringen Volumen an geeignetem Lösungsmittel aufgenommen werden und anschließend am MCR gemessen werden. Vorteile der IMS sind die Aufkonzentrierung der Analyten und gleichzeitige Eliminierung von Matrixbestandteilen, welche den Immunoassay negativ beeinflussen können. Um die immunomagnetischen Nanopartikel zu syntheti- 
sieren wird zunächst Eisen-oleat aus Eisen-(III)chlorid und Natriumoleat hergestellt. Anschließend werden auf der Oberfläche von Silica-Nanopartikeln Eisen-Nanopartikel durch thermische Dekomposition des Eisen-oleats hergestellt. ${ }^{[8]}$ Die resultierenden Silica-Kern Eisenoxid-Schale Nanoverbundstrukturen werden nach geeigneter Aufreinigung mit GOPTS und Jeffamine funktionalisiert und anschließend können Antikörper an die Nanoverbundstrukturen gebunden werden.

\section{Ergebnisse}

MC-LR konnte erfolgreich auf der MicroarrayOberfläche immobilisiert werden. Nach Optimierung von Lösungsmittelzusammensetzung, $\mathrm{pH}$ und $\mathrm{MC}$ LR Konzentration der Immobilisationslösung von MC-LR bezogen auf die höchstmögliche Signalintensität wurden Kalibrierungen durchgeführt. Die Kalibrierung ist ein Triplikat von drei Kalibrierungen auf drei unterschiedlichen Microarrays gemessen in Leitungswasser. Diese Versuche zeigten eine Nachweisgrenze für MC-LR von 4,8 $\mu \mathrm{g} / \mathrm{L}$, einen Arbeitsbereich von $5,87 \mu \mathrm{g} / \mathrm{L}-36,09 \mu \mathrm{g} / \mathrm{L}$ und einen $\mathrm{IC}_{50}$ von $14.56 \mu \mathrm{g} / \mathrm{L}$. Die Kalibrierkurve ist in Abb. 4 dar-

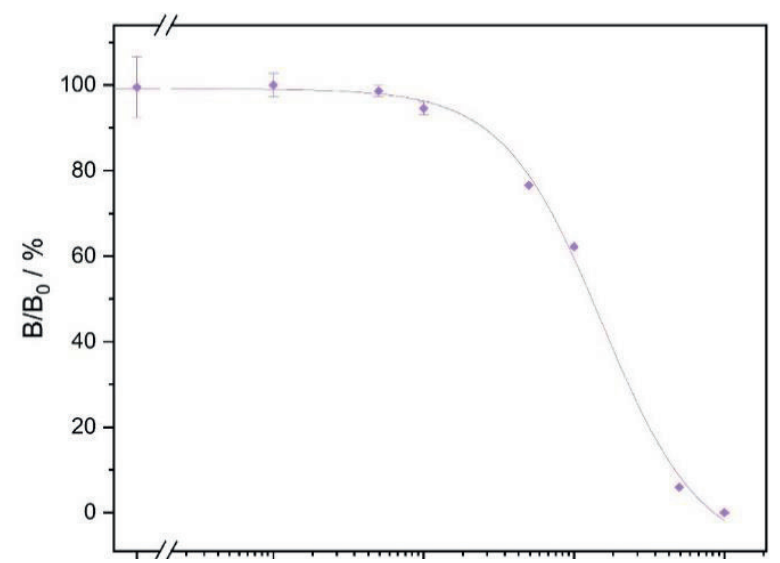

Abb. 4: Kalibrierkurve von MC-LR.

gestellt.

Im Weiteren sollen Wiederfindungsexperimente durchgeführt werden und Effekte von Realmatrizes auf den Immunoassay untersucht werden. Außerdem sollen Realproben von Seen untersucht werden, bei denen Algenblüten festgestellt wurden und falls MC-LR nachgewiesen werden kann mit einer Referenzmethode wie zu Beispiel HPLC bestätigt werden.

Für die Aufkonzentrierung der Cyanotoxine mittels IMS konnten die magnetischen Nanopartikel erfolgreich hergestellt werden. REM-Aufnahmen haben bestätigt, dass die Eisenoxid-Nanopartikel gleichmäßig auf der Oberfläche der Silica-Nanopartikel verteilt sind. Die Anwesenheit von Eisen nach der Nanopartikelsynthese wurde anhand von EDXMessungen bestätigt und vorläufige Tests haben gezeigt, dass das Binden der Antikörper an die magnetischen Nanopartikel erfolgreich durchgeführt werden konnte. Im nächsten Schritt wird der Immunoassay für die Messung der immunomagnetischen Nanopartikel am MCR entwickelt und optimiert. Anschließend wird eine Methode entwickelt, um die Aufkonzentrierung der Cyanotoxine mittels IMS zu automatisieren.

\section{Diskussion und Ausblick}

Das Online-Monitoring-System soll parallel Daten aller relevanter Parameter für das Algenmonitoring sammeln, damit eine selbstlernende künstliche Intelligenz Vorhersagemodelle für ein übermäßiges Wachstum von Cyanobakterien erstellen kann, indem Echtzeitdaten mit Hilfe eines Cloudbasierten Datenmanagement-Systems ausgewertet werden. Im ersten Schritt werden gesammelte Oberflächenwasserproben im Labor analysiert, damit erste Daten des Sensorsystems in die Cloud transferiert und mit dem Algorithmus von AUG ausgewertet werden können. Im Anschluss werden im Feld Echtzeitdaten aus Oberflächenwasser mit dem Sensorsystem erfasst. Um in den Wintermonaten eine wesentliche Änderung der Nitratkonzentrationen im Oberflächenwasser zu simulieren, werden dafür neben den online-Feldmessungen Proben gesammelt und im Labor künstlich mit Nitrat versetzt. Anschließend werden die Nitratkonzentrationen mit dem optischen Sensormodul bestimmt. Im Weiteren sollen Feldmessungen an Gewässern durchgeführt werden, bei denen bekannt ist, dass in den Sommermonaten Algenblüten auftreten.

Parallel soll eine Messmethode für die immunomagnetischen Nanopartikel am MCR-R weiterentwickelt und die immunomagnetische Separation automatisiert werden. Es soll möglich sein Cyanotoxine aus dem Oberflächenwasser vollautomatisch an die immunomagnetischen Nanopartikel anzureichern und anschließend die Nanopartikel zu extrahieren, um deren Beladung mit MC-LR zu messen.

Somit soll ein Frühwarnsystem entstehen, welches in der Lage ist Oberflächenwasser kontinuierlich zu überwachen und Algenblüten anhand der Beobachtung bestimmter Parameter frühzeitig voraussagen zu können. Bei signifikanten Veränderungen von Schlüsselparametern, kann das Biosensorsystem MCR-R den Anstieg von Cyanotoxinen bestätigen. Somit kann frühzeitig gewarnt werden, wenn eine Gefahr für Mensch oder Tier besteht.

\section{Literatur}

1. Moore, S.K., et al. Impacts of climate variability and future climate change on harmful algal blooms and human health. in Environmental health. 2008. Springer. 
2. Badewasserrichtlinie (EG-BadewRL) Richtlinie 2006/7/EG des Europäischen Parlaments und des Rates vom 15 Februar 2006 über die Qualität von Badegewässern und dere Bewirtschaftung und zur Aufhebung der Richtlinie 76/160EWG.

3. Krueger, F. (2021, 3. Juni). Hund verendet Sorge wegen Blaualgen im Tegeler See. rbb24.

https://www.rbb24.de/panorama/beitrag/202 1/06/tegeler-see-berlin-tegel-blaualgen.html.

4. Lauer, M. (2021, 23. Juli). Blaualgen: Hund stirbt nach Bad im Mandichosee. br. https://www.br.de/nachrichten/bayern/blaual gen-hund-stirbt-nach-bad-immandichosee, SduKzxp.

5. Butie, L., (2021, 30. Juli). Fünf Hunde sterben wegen Blaualgen. 20min. https://20min.ch/story/fuenf-hunde-sterbenwegen-blaualgen-396831180059.

6. Huebner, M., et al., Layer-by-layer generation of PEG-based regenerable immunosensing surfaces for small-sized analytes. Biosensors and Bioelectronics, 2015. 67: p. 334-341.

7. Zeck, A., et al., Generic microcystin immunoassay based on monoclonal antibodies against Adda. Analyst, 2001. 126(11): p. 2002-2007.

8. Nistler, A., et al., Production and characterization of long-term stable superparamagnetic iron oxide-shell silicacore nanocomposites. Journal of Magnetism and Magnetic Materials, 2017. 442: p. 497503. 